\title{
Les mutations de l'action internationale des régions françaises
}

\author{
Christophe Mestre ${ }^{a}$
}

RÉSUMÉ. L'action extérieure des collectivités territoriales françaises est une politique ancienne, bien ancrée. Les régions, érigées en 1982, sont les plus jeunes collectivités territoriales françaises. Dès leur création, elles ont développé une action internationale ambitieuse, marquée par des relations de coopération décentralisée au développement. Depuis 2016, on constate un tournant des politiques régionales d'internationalisation qui passent de la solidarité internationale vers l'intérêt pro domo: priorité à l'attractivité, au rayonnement et au développement économique, changement de géographie d’intervention vers les pays émergents, arrêt de relations avec des collectivités territoriales des pays en voie de développement. Selon plusieurs observateurs, ces évolutions s'expliquent par les contraintes budgétaires, le changement générationnel des élus, la perception d'une demande sociale vers un retour sur le territoire français. Elles posent surtout la question du rôle des régions comme acteurs centraux pour contribuer à répondre aux défis planétaires que sont le climat, les migrations, la sécurité, la lutte contre les inégalités et le développement.

\begin{abstract}
The external action of French regional authorities is an old and well-established policy. The Régions, established in 1982, are the youngest French regional authority entities. Upon their creation, they developed ambitious international action, marked by decentralized cooperation development relations. Since 2016, there has been a turning point in internationalization regional policies that are moving from international solidarity to pro-domo interest: priority for economic regional attractiveness, development and influence, shifting intervention towards emerging countries and ceasing relations with regional authorities in developing countries. According to numerous observers, these evolutions may be explained by financial constraints, the generational change in elected representatives and the perceived social requirement for returning to the French regions. It mainly questions the role of the regions as main actors to contribute in meeting the world challenges in the fields of climate, migrations, security, fight against inequality and development.
\end{abstract}

\section{Introduction}

L'action internationale ou extérieure des collectivités territoriales françaises, depuis les années 1980, a été marquée par la prééminence de la coopération décentralisée au développement, certaines régions françaises pouvant maintenir des relations de coopération décentralisée au développement avec plus d'une dizaine de régions des pays du Sud.

Depuis la mise en place de la nouvelle carte régionale et des nouveaux exécutifs régionaux en janvier 2016, cette politique régionale de solidarité internationale semble en recul au profit d'une politique d'attractivité régionale visant le développement économique du territoire régional français. Qu'en est-il réellement?
Assiste-t-on à un changement d'orientation des politiques régionales et si oui pourquoi?

Le présent article après avoir présenté le cadre de l'action extérieure des collectivités territoriales françaises et la place de la coopération décentralisée au développement dans celle-ci s'intéresse à la dimension régionale de l'action extérieure des collectivités territoriales. Partant de l'histoire des régions françaises et du développement rapide de leurs relations internationales, l'article montre les évolutions survenues à partir des dernières élections régionales de 2015 et en analyse les raisons. Enfin, la conclusion évoque les perspectives de l'action extérieure des collectivités territoriales.

a Enseignant chercheur, CIEDEL (Centre international d'études pour le développement local, Université Catholique de Lyon) 
Cet article s'inscrit dans le cadre d'une recherche menée par le CIEDEL (Centre International d'Études pour le Développement Local) et CUF (Cités Unies France) sur l'internationalisation des villes et des territoires.

\section{L'action internationale des collectivités territoriales françaises, une histoire longue marquée par l'expansion de la coopération décentralisée au développement}

Contrairement à une idée reçue, l'action internationale des collectivités territoriales françaises n'est pas un phénomène nouveau, mais au contraire s'inscrit dans la longue durée. Historiquement en relation avec d'autres collectivités territoriales dès le XIXe siècle plus précisément en 1913, elles participent, en marge de l'exposition universelle, au congrès international des villes à Gand qui donna naissance au premier réseau international des villes, l’Union Internationale des Villes (UIV).

Cette action internationale visait alors à échanger entre villes sur les modèles de gouvernements urbains, mais aussi à avoir plus de poids sur le plan politique d'un point de vue international (ex. demande d'une implication dans les espaces politiques de la Société des Nations - SDN).

C'est après la Seconde Guerre mondiale, avec le développement des jumelages que l'action internationale des collectivités territoriales françaises a littéralement explosée : jumelages intra-européens (Allemagne, Italie...), pour tisser des liens entre les peuples afin que l'on ne revive "plus jamais ça »; puis se sont développés les jumelages avec des communes des pays frères (URSS et pays de l'Est $\left.^{1} \ldots\right)$; enfin dès les indépendances des jumelages avec les grandes villes des jeunes États africains (ex. jumelage Reims Brazzaville en 1961) (France Diplomatie, 2018).

Ces relations avec des pays du Sud se sont ensuite développées avec le contrecoup des évènements dramatiques vécus dans les pays du Sahel dans les années 1970 et 1980 (grande sécheresse de 1973, puis celle de 1984), avec le développement des phénomènes migratoires (relations avec les communes marocaines, tunisiennes, maliennes...) et plus récemment avec les mouvements de démocratisation et de décentralisation de la décade des années 1990 tant en Afrique de l'Ouest et centrale (Burkina $\mathrm{FasO}^{2}$, Sénégal...), que dans certains pays de l'Est (Roumanie ${ }^{3}$ ) ou des Caraïbes (Haïti).

Longtemps marqués par un caractère humanitaire qui leur a valu le nom de " coopération container $»^{4}$ (Husson et Mestre, 2012), ces jumelages ont évolué ensuite vers ce qu'il est convenu d'appeler la coopération décentralisée au développement. Ils ont ainsi épousé les évolutions des démarches de l'aide au développement en passant de l'appui aux services sociaux de base (le puits, le dispensaire, l'école), aux projets de développement local (basé sur la gestion de fonds d'investissement et la planification locale) à des actions d'appui institutionnel aux nouvelles collectivités territoriales des pays en développement (renforcement des capacités, mise en place de services techniques, recouvrement fiscal...). Enfin, certains se sont transformés en de véritables actions de renforcement de la diplomatie des villes en introduisant leurs villes partenaires dans les espaces internationaux de négociation de l'agenda politique international et de l'agenda du développement.

Parallèlement, les collectivités territoriales françaises, en particulier les grandes villes (par exemple Paris, Bordeaux, Lyon), les départements (par exemple l'Aude, l'Essonne, le Nord) et les régions (par exemple Rhône-Alpes; la Picardie, Nord Pas de Calais) ont densifié leur action internationale qui s'est peu à peu diversifiée :

- appui à la mobilité entrante et sortante des étudiants et des apprentis;

- ouverture à la programmation culturelle internationale;

- internationalisation dans le domaine du sport;

- promotion économique de leur territoire et de leurs entreprises (missions conjointes élus et chefs d'entreprises, salons...);

- éducation au développement et à la citoyenneté internationale en milieu scolaire et grand public;

- subvention aux actions de solidarité internationale des acteurs des territoires (Associations, $\mathrm{ONG}, \ldots)$;

- implication ou constitution de réseaux généralistes ou spécialisés de collectivités territoriales (Cités et gouvernement locaux unis, réseau international des villes créatives, club des plus belles baies du monde...); 
- implication dans les agendas et négociations internationales (Habitat II, Conférences des parties sur le climat...).

Cette évolution et cette densification des relations internationales des collectivités territoriales françaises s'est faite à partir d'un cadre juridique souple et évolutif, qui entérine des situations déjà existantes :

- Un décret de $1956^{5}$ qui donne un cadre juridique aux jumelages (Vion, 2003);

- La Loi no 92-125 relative à l'administration territoriale de la République du 6 février 1992 qui autorise les collectivités françaises à conclure des conventions avec les collectivités étrangères dans le respect des engagements internationaux de la France;

- La Loi no 2007-147 du 2 février 2007 relative à l'action extérieure des collectivités territoriales et de leurs groupements (dite loi Thiollière) qui permet aux collectivités territoriales d'intervenir en cas d'urgence avec des territoires avec lesquelles elles n'ont pas de convention de coopération;

- La Loi no 2014-773 du 7 juillet 2014 d'orientation et de programmation relative à la politique de développement et de solidarité internationale (dite loi Canfin) qui permet aux collectivités territoriales de « mettre en œuvre ou soutenir toute action internationale annuelle ou pluriannuelle de coopération, d'aide au développement ou à caractère humanitaire » sans que cela se fasse obligatoirement dans le cadre de conventions ॥; - La Loi no 2016-1657 du 5 décembre 2016 relative à l'action extérieure des collectivités territoriales et à la coopération des outre-mer dans leur environnement régional (dite loi Letchimy) qui permet aux collectivités territoriales de passer des conventions avec des États étrangers.

Cet arsenal de textes s'inscrit dans un cadre législatif plus large permettant aux collectivités territoriales et aux établissements publics de coopération intercommunaux de consacrer jusqu'à $1 \%$ de leur budget des services de l'eau, de l'assainissement, de l'énergie, des déchets et sans doute demain des transports à des actions de solidarité internationale.

L'évolution de ce cadre juridique va d'une part dans le sens de reconnaittre la compétence internationale des collectivités territoriales, mais en même temps supprime la nécessité de l'inscrire dans un cadre conventionnel en permettant aux collectivités d'agir de manière ponctuelle dans tous les pays comme peut le faire une organisation non gouvernementale (ONG).

On compte aujourd'hui près de 5000 collectivités territoriales françaises (sur un peu plus de 36000 , soit près de $15 \%$ ) qui ont des partenariats avec plus de 8000 collectivités territoriales étrangères dans plus de 130 pays (Ministère des Affaires étrangères et européennes, 2019b). Ces collectivités territoriales françaises mobilisent environ 1 milliard pour leurs actions internationales dont une centaine de millions pour la coopération décentralisée au développement (Ministère des Affaires étrangères et européennes, 2019a).

Cette densification des actions de coopération décentralisée au développement s'est accomplie jusqu'au milieu de la présente décennie, où les élections municipales de 2014, puis régionales de 2015 ont marqué une évolution nette de ces orientations.

\section{Les régions françaises : construction récente, action internationale dynamique (1982 - 2016)}

Après l'échec de la régionalisation promue par le Général de Gaulle en 1969, les régions françaises sont les plus jeunes collectivités territoriales de la République. Erigées en collectivités territoriales en 1982, elles ont pour mission de contribuer au développement économique, social et culturel du territoire régional.

À ce titre, la majeure partie des régions françaises se sont inscrites, dès leur création, dans une action internationale ambitieuse. Elles ont noué des relations internationales intra-européennes, des relations de coopération transfrontalière, se sont impliquées ou ont créé des réseaux de collectivités territoriales et bien entendu ont développé des relations de coopération décentralisée au développement en particulier avec les pays dont les processus de décentralisation mettait en avant le niveau régional (Mali, Sénégal, Madagascar) ou permettait le développement d'intercommunalités à vocation de préfiguration d'un échelon de type régional (Bénin par exemple). Elles ont également développé de nombreuses relations sur des géographies plus lointaines, que ce soit en Asie (Laos, Vietnam), 
en Amérique Latine (Argentine, Brésil) ou au Moyen-Orient (Liban, territoires Palestiniens).

Disposant de marges de manœuvre budgétaires, de services techniques dédiés à l'international, d'un tissu d'opérateurs régionaux compétents et engagés, les régions françaises se sont engagées très rapidement dans de nombreuses relations de coopération décentralisée au développement. Au fur et à mesure de la succession des mandats des élus régionaux et des changements des élus régionaux responsables de l'action internationale, les géographies des relations internationales des régions françaises, extrêmement sollicitées par les partenaires potentiels, se sont diversifiées rendant les politiques internationales régionales difficilement compréhensibles avec la tendance à disperser les moyens et à déléguer ces actions et relations à des opérateurs techniques $(\mathrm{ONG}$, associations, bureaux d'étude) en lieu et place d'une implication des services.

Cette «boulimie» relationnelle a amené, dans certains cas, à une concurrence régionale. Certaines régions partenaires (par exemple la région aujourd'hui disparue de Saint-Louis au Sénégal) allant jusqu'à «collectionner» les relations avec des Régions françaises profitant ainsi de leur attrait historique, touristique et symbolique et du charisme de leurs responsables techniques et politiques.

Indéniablement, le développement des relations internationales des régions françaises leur a permis de renforcer leur place à l'international, les positionnant comme des acteurs clefs dans les négociations internationales, en particulier par le biais des réseaux internationaux de collectivités territoriales (Cités et gouvernements locaux unis, Organisation des régions unies, Association régionale des régions francophones...).

\section{L'année 2016, un tournant de l'action internationale régionale : de la solidarité à l'intérêt pro domo?}

Les élections municipales françaises de 2014 puis les élections régionales de fin 2015 ont vu un important changement des majorités au sein des gouvernements locaux. Sur le plan régional, alors qu'en 2010, 22 des 25 régions étaient de gauche en 2015, ce sont 7 des 17 nouvelles régions dont la majorité reste à gauche, 8 ayant une majorité de droite et 2 , une majorité régionaliste.

De manière concomitante, au premier janvier 2016, la Loi no 2015-991 portant sur la nouvelle organisation territoriale de la République (dite loi NOTRe) est entrée en vigueur avec un redécoupage territorial de la plupart des régions métropolitaines qui sont le produit de la fusion de plusieurs régions, ainsi qu'avec une extension des compétences régionales ${ }^{6}$, sans pour autant que celles-ci ne voient leur budget augmenter de manière substantielle.

Ces évolutions, politiques, territoriales, budgétaires et aussi de leurs missions ont amené les régions à faire un réel travail de relecture de leurs politiques publiques régionales et de redéfinition des priorités budgétaires. Cette relecture des politiques publiques a également touché la politique internationale et en particulier la politique de coopération décentralisée au développement.

En conséquence on a constaté une évolution des politiques internationales régionales, qu'il faut nuancer en fonction des particularités régionales, mais qui globalement va dans le sens suivant :

Le développement d'une politique d'attractivité et de rayonnement visant le développement économique régional

- La plupart des régions mettent en avant de leur politique internationale le rayonnement et l'attractivité de leur territoire et utilisent pour se faire les techniques du marketing territorial. À titre d'exemple, la région Normandie, issue de la fusion des régions Basse et Haute-Normandie a développé le label «Normandie pour la Paix » qui vise à renforcer une image internationale de la Normandie et à développer le tourisme de mémoire;

- Ces politiques d'attractivité et de rayonnement visent le développement économique régional et en particulier le développement de l'emploi en région;

- L'action internationale des régions est centrée sur la recherche d'un intérêt local, qui est l'intérêt économique du territoire régional. La recherche de cet intérêt local peut être mutuel avec le ou les territoires partenaire(s), ou, et c'est le plus souvent le cas, un intérêt pro domo, sur l'intérêt pour la région française. 


\section{La diversification des modalités d'actions internationales}

- Les régions passent de relations conventionnelles avec des territoires régionaux partenaires à des relations ponctuelles, limitées dans le temps, par exemple dans le cadre de l'organisation de missions de délégation composée d'élus et d'entrepreneurs dans des pays émergents ou à revenus intermédiaires (Russie, Japon, Corée, Chine, Inde...);

- Les régions développent aussi des dispositifs visant à favoriser la mobilité entrante et sortante en particulier pour les étudiants. Ceci d'une part afin d'ouvrir le territoire régional français à l'international et d'autre part pour que les étudiants étrangers repartent avec une bonne image du territoire régional français et en deviennent les ambassadeurs;

- Les régions mobilisent les acteurs du territoire pour organiser salons, missions... valorisant les atouts et l'offre technique, industrielle, commerciale de leur territoire auprès des partenaires étrangers ${ }^{7}$;

- Les régions développent les actions de promotion touristique de leur territoire, que ce soit pour le tourisme gastronomique, culturel, patrimonial, ou sportif;

- Enfin, elles favorisent le maillage interpersonnel, interinstitutionnel pour développer des liens de longue durée entre acteurs des territoires en relation.

\section{Le changement des géographies d'intervention}

- Les géographies des pays d'intervention évoluent avec un tropisme fort des régions françaises vers les pays à revenu intermédiaire et les pays émergents ou perçus comme tels. À ce titre, les pays en développement, à l'exception des pays comme la Côte d'Ivoire ne sont plus considérés comme prioritaires, et les pays structurellement déficitaires ou en crise comme le Niger ou Haïti n'attirent plus de nouvelles coopérations;

- Ce changement de géographie des pays d'intervention est renforcé par la problématique de l'insécurité dans la bande sahélo saharienne qui renforce les difficultés d'une relation viable entre acteurs des collectivités partenaires (interdiction de déplacement des représentants des collectivités territoriales françaises dans les zones rouges);

- La situation d'insécurité, les retards dans les processus électoraux nationaux (par exemple au
Togo, à Madagascar, au Niger...) ainsi que les changements dans l'architecture institutionnelle (par exemple au Sénégal avec la suppression des régions) amènent certaines régions françaises à suspendre voire à arrêter des relations de coopération décentralisée parfois fort anciennes.

\section{Le changement au sein des services internationaux des régions}

- Les services dédiés aux actions internationales voient leurs noms changer, devenant par exemple des « directions de l'attractivité ». Ils voient aussi leur rattachement évoluer pour être de plus en plus intégrés dans les directions de l'économie;

- Ces changements, qui ne sont pas uniquement sémantiques ou techniques, amènent à un réel repositionnement tant des services que des agents de la collectivité territoriale régionale qui doivent apprendre de nouveaux métiers et travailler avec de nouveaux acteurs.

\section{L'arrêt d'une partie des relations de coopération décentralisée au développement}

- Si certaines régions ont mis fin à leurs relations de coopération décentralisée au développement, la majeure partie de celles-ci les ont surtout réduites, soit en nombre, soit sur le plan du budget alloué et en importance institutionnelle. La coopération décentralisée au développement n'est plus alors qu'une des modalités parmi d'autres de l'action internationale des régions, et n'est plus mise en avant comme marqueur de l'action internationale régionale;

- Ces ruptures ont souvent été réalisées de manière unilatérale, sans négociation avec les collectivités partenaires et sans respecter les conventions existantes entre les régions, voire en remettant en cause le déroulement même de projets en cours. Ce fut le cas de la région Ile de France qui a interrompu les programmes en cours, parfois sur financement externe, avec les instituts d'urbanisme mis en place à Madagascar ou au Viêt Nam.

En résumé, les régions maintiennent, sous différentes formes, des actions de solidarité internationale, mais réduites, et substituent souvent des pratiques de coopération décentralisée au développement en appui direct aux régions partenaires (appui institutionnel, aide budgétaire...) par des subventions à des opérateurs (ONG, bureaux 
d'étude), gommant souvent la spécificité que représente la coopération décentralisée au développement. Ceci est dommage, car cette coopération entre pairs permettait :

- l'implication de services techniques et les échanges entre ceux-ci;

- une aide budgétaire ou sur projet à travers des transferts financiers de trésor public à trésor public renforçant ainsi les administrations nationales;

- une inscription des investissements financés par la coopération dans les budgets régionaux, contribuant à une réelle maîtrise d'ouvrage régionale.

L'appui institutionnel aux collectivités partenaires devient par conséquent l'exception, au bénéfice de subventions par projet à des opérateurs de la coopération décentralisée au développement. Cette évolution pose de réelles questions sur l'objet de cet appui, et sur son intérêt pour la collectivité territoriale partenaire qui n'est pas toujours en capacité d'absorber de nouveaux flux de coopération si son fonctionnement n'est pas assuré sur le plan financier, ce qui est souvent le cas, faute de ressources pour disposer des équipes techniques suffisantes, pour assurer la prise en charge des frais de fonctionnement courant (communication, déplacement...) et pour renforcer sa capacité à collecter des ressources propres (fiscalité, taxe, produits des services et des équipements...) pour atteindre la viabilité à moyen terme.

\section{Les raisons du désenchantement vis-à-vis de la coopération décentralisée au développement}

Cette inflexion de l'action internationale des régions vers la recherche d'un intérêt pro domo ramenant les politiques de solidarité internationale et donc de coopération décentralisée au développement comme étant une des politiques parmi d'autre s'explique par un faisceau de raisons, dont les principales sont les contraintes budgétaires, le changement générationnel des élus et la perception d'une demande sociale de résultats pour la région:

\subsection{Les contraintes budgétaires}

Les évolutions institutionnelles (fusion des régions, élargissement des compétences) et budgétaires (limitation des transferts de l'État, etc.) ont amené toutes les régions à revoir leurs affectations budgétaires et à réduire les dépenses dans tous les secteurs, mais en particulier dans les compétences non obligatoires ${ }^{8}$ et dans les secteurs jugés non rentables ou à rentabilité différée.

Ces réductions budgétaires ont affecté fortement les politiques de solidarité internationale, et donc la coopération décentralisée au développement, dont les budgets ont diminué de moitié ces dernières années dans certaines régions.

\subsection{Le changement générationnel d'élus}

Les changements politiques au sein des exécutifs et délibératifs ne sont pas obligatoirement significatifs en termes de changement d'orientation en matière de coopération décentralisée, la coopération décentralisée faisant jusqu'ici l'objet d'une certaine unanimité politique.

Par contre, on constate un changement de génération d'élus. La génération des élus qui a vécu les processus des indépendances des pays du Maghreb et d'Afrique, et qui a participé aux actions de la coopération au développement comme coopérants, a été remplacée progressivement par des élus plus jeunes qui n'ont pas cette culture de coopération, ni le sentiment d'une «dette» morale envers les pays en développement.

Pour beaucoup d'entre eux, ces nouveaux élus ont une sensibilité plus ténue à la solidarité internationale, à la coopération décentralisée au développement et voient plus difficilement les raisons qui pousseraient les collectivités régionales à s'engager dans ce champ qui est déjà pris en charge par l'État et par les ONG.

\subsection{La perception d'une demande sociale de résultats pour la région}

Les élus, et en particulier les nouveaux élus, partagent la perception que leurs électeurs ne sont pas sensibles à la solidarité internationale et donc qu'ils ne soutiennent pas l'engagement de la collectivité régionale dans des relations de coopération décentralisée au développement. Les élus ont une perception comme quoi la demande sociale vis-à-vis de la région est de répondre au défi principal des citoyens qui est celui de l'emploi et du pouvoir d'achat. La solidarité internationale, les enjeux planétaires (climat, migrations, sécurité, développement, environnement...) sont ainsi vus comme ne relevant pas du champ d'action prioritaire des régions. 
En résumé, derrière toutes ces raisons, il convient de se poser la question de savoir s'il n'y a pas un changement plus profond pour ce qui est des référentiels des décideurs, du système de valeurs des élus eux-mêmes?

En effet, nous sommes dans une période ou l'internationalisation des territoires n'a jamais été aussi grande, ou l'interdépendance des territoires n'a jamais paru si évidente (climat, sécurité, développement n'ont pas ou plus de frontières!). Dans le même temps, les régions réduisent leur contribution aux politiques publiques locales qui participent au rééquilibrage entre pays développés et pays sous-développés au bénéfice de politiques visant à créer de la richesse et de l'emploi en France. N'estce pas tout simplement le signe d'une évolution des valeurs des décideurs politiques où les valeurs de compétitivité, d'intérêt local sont plus importantes que les valeurs de solidarité, de redistribution?

On constate en tous les cas que cette question des référentiels se pose avec acuité au sein des exécutifs des régions. En effet, les responsabilités internationales sont de plus en plus réparties entre un nombre important de conseillers régionaux sans que ceux-ci ne partagent d'orientations communes, amenant parfois à une certaine confusion sur les orientations et les motivations profondes de la collectivité régionale.

\section{Quel avenir pour l'action internationale des régions?}

En France, le poids politique, économique et social des régions est de plus en plus important. Il permet à celles-ci de constituer potentiellement un réel «contrepouvoir» face à l'État. Par ailleurs les régions, par leur taille (la région Auvergne RhôneAlpes a plus de 8 millions d'habitants, soit autant que la Suisse), par leur faible nombre (13 régions métropolitaines) ont un rôle essentiel à jouer à l'international. Certains responsables des régions ont bien compris que la coopération internationale est l'un des axes clefs de leur développement.

Toutefois, le recentrage de l'action régionale vers l'attractivité, le rayonnement, le développement économique dans une perspective de développement de leur territoire gomme d'une part, la responsabilité, et d'autre part, la capacité des territoires régionaux à contribuer à résoudre les défis planétaires auxquels nous sommes confrontés (climat, migration, sécurité, développement, lutte contre les inégalités, emploi des jeunes, etc.) ainsi qu'à contribuer à rééquilibrer la répartition des richesses à l'international, ce qui est l'une des conditions sine qua non de la paix et du développement à l'échelle planétaire.

Or la nécessité de l'aide au développement pour l'avenir de notre planète est une conviction partagée par un nombre de plus en plus important de citoyens français. Les baromètres de l'Agence française de développement montrent que $70 \%$ des Français sont favorables à un soutien au pays en développement. Cette statistique ne semble pas vouloir diminuer malgré les tensions économiques et migratoires que connait la France depuis quelques années (Agence française de développement, 2018).

Il y a donc un réel enjeu de contribuer à repenser le rôle des régions françaises en matière de développement international et leur contribution au développement à l'échelle mondiale.

Les acteurs des territoires régionaux ont donc à se mobiliser pour plaider auprès de leurs élus pour que la solidarité internationale et la coopération au développement international soient pleinement intégrées aux politiques internationales des régions.

\section{NOTES}

1 Par exemple jumelage de Rennes avec Brno en République Tchèque dès 1954.

2 Au Burkina Faso, ce sont les deux tiers des 351 communes du pays qui ont des relations de coopération décentralisée au développement avec des collectivités territoriales européennes (Ministère des Affaires étrangères et européennes, 2011).

3 À la fin de la période Ceausescu, alors que le pouvoir en place planifiait la « systématisation du territoire » passant par la destruction de l'habitat traditionnel roumain, une dynamique de soutien avec le peuple roumain s'est développée en France sous l'égide d'OVR (Opération Villages Roumains), qui a vu des centaines de communes françaises se mobiliser en solidarité avec les villages roumains, ce qui a débouché après la révolution sur la mise en place de très nombreux jumelages. 
4 Les premières actions de coopération des comités de jumelages ont longtemps été marquées par un caractère caritatif basé sur l'envoi par les collectivités territoriales françaises de dons d'objets ou de matériels français usagés par container, d'où le nom de cette coopération, la « coopération container ».

5 Décret du 24 janvier 1956 portant création d'une commission chargée de coordonner les échanges internationaux dans le domaine communal.

6 La loi NOTRe précise : «Le conseil régional a compétence pour promouvoir le développement économique, social, sanitaire, culturel et scientifique de la région, le soutien à l'accès au logement et à l'amélioration de l'habitat, le soutien à la politique de la ville et à la rénovation urbaine et le soutien aux politiques d'éducation et l'aménagement et l'égalité de ses territoires, ainsi que pour assurer la préservation de son identité et la promotion des langues régionales, dans le respect de l'intégrité, de l'autonomie et des attributions des départements et des communes».

7 Par exemple la Région Auvergne Rhône-Alpes soutien l'organisation du salon «Pollutec » qui se veut l'événement de référence des professionnels de tous les secteurs de l'environnement, qu'ils soient engagés dans sa préservation ou contraints par des pollutions existantes ou le manque d'accès à des ressources. En 2018, ce sont des représentants de128 pays qui y ont participé. En particulier la Région Auvergne Rhône-Alpes soutien la participation de délégations étrangères au salon.

8 En France les collectivités territoriales ont des compétences obligatoires, qu'elles doivent assumer et des compétences facultatives qu'elles peuvent assumer ou non en fonction des choix politiques des délibératifs.

\section{RÉFÉRENCES}

Agence française de développement (2018). Baromètre de l'AFD : $70 \%$ des français favorables à un soutien aux pays en développement. Repéré à https://www.afd.fr/fr/barometre-de-lafd-70-des-francais-favorables-un-soutien-aux-pays-endeveloppement

France Diplomatie (2018). L'action extérieure des collectivités territoriales. Repéré à https://www.diplomatie.gouv.fr/fr/ politique-etrangere-de-la-france/action-exterieure-des-collectivites-territoriales/presentation-et-activites-de-la-cncd-et-dela-daect/

Husson, B. et Mestre, C. (2012). La coopération décentralisée, aide, solidarité, appui ou coopération. Leçon de l’analyse des pratiques. Cahiers de la coopération décentralisée, 3.

Ministère des Affaires étrangères et européennes (2010). La coopération décentralisée entre l'Afrique et la France. 50 ans de dialogues et d'échanges pour le développement [Brochure]. Repéré à http://www.bfcinternational.org/IMG/pdf/Brochure200x270mmBD_1_.pdf

Ministère des Affaires étrangères et européennes (2011). La coopération décentralisée au Burkina Faso. Évaluation no 116. Repéré à https://www.oecd.org/countries/burkinafaso/48467711.pdf

Ministère des Affaires étrangères et européennes (2019a). Les chiffres clés de l'AECT. Repéré à https://www.diplomatie.gouv.fr/fr/politique-etrangere-de-la-france/action-exterieure-des-collectivites-territoriales/les-chiffres-cles-de-l-aect/

Ministère de l'Europe et des Affaires étrangères (2019b). L'action extérieure des collectivités territoriales. Repéré à https://www.diplomatie.gouv.fr/fr/politique-etrangere-de-la-france/action-exterieure-des-collectivites-territoriales

Vion, A. (2003). L'invention de la tradition des jumelages (1951-1956) : mobilisations pour un droit. Revue française de science politique, 53(4), 559-582. doi.org/10.3917/rfsp.534.0559 